\title{
LecT-Hepa facilitates estimating treatment outcome during interferon therapy in chronic hepatitis $C$ patients
}

\author{
Xia Zou ${ }^{1,4,6+}$, Xiumei Chi ${ }^{2 \dagger}$, Yu Pan², Dongning Du ${ }^{3,4}$, Haibo Sun ${ }^{2}$, Atsushi Matsuda ${ }^{3,4}$, Wei Li ${ }^{1,4}$, Atsushi Kuno ${ }^{3,4}$, \\ Xinxin Zhang ${ }^{5}$, Hisashi Narimatsu ${ }^{3,4}$, Junqi Niu ${ }^{2^{*}}$ and Yan Zhang ${ }^{1,4^{*}}$
}

\begin{abstract}
Background: A combination treatment of interferon and ribavirin is the standard and the commonly used treatment for chronic hepatitis $\mathrm{C}(\mathrm{CHC})$. Developing noninvasive tests like serum indicators that can predict treatment outcome at an early stage of therapy is beneficial for individualized treatment and management of $\mathrm{CHC}$. A glyco-indicator based on the glyco-alteration of serum a1-acid glycoprotein, LecT-Hepa, was discovered by glycomics technologies as a robust indicator of liver fibrosis. Here, we investigated the clinical utility of LecT-Hepa for evaluation of treatment outcome.

Results: Firstly, ninety-seven patients with CHC were used for comparison of LecT-Hepa in serum and plasma. We found no significant difference in the concentrations of LecT-Hepa in serum and plasma. And then, 213 serum specimens from 45 patients who received 48 weeks of treatment with interferon and ribavirin were followed up for 96 weeks, and were used for evaluation of the role of LecT-Hepa. We found that LecT-Hepa might reflect the change in fibrosis regression during the treatment process. Moreover, the change of LecT-Hepa at the first 12 weeks of treatment could already predict the antiviral treatment response, which was more superior to FlB-4 index and aspartate aminotransferase-to-platelet ratio index (APRI) in this study.
\end{abstract}

Conclusions: These results provide a new perspective that serum glycoprotein could be used as a joint diagnosis indicator for estimation treatment outcome of viral hepatitis at earlier stage of therapy.

Keywords: Glycoprotein, LecT-Hepa, Non-invasive, Treatment outcome, CHC

\section{Introduction}

Chronic hepatitis $\mathrm{C}$ virus (HCV) infection is a highly prevalent public health concern and one of the leading causes of cirrhosis, hepatocellular carcinoma, and liver failure [1]. An estimated 150 million people worldwide are chronically infected with $\mathrm{HCV}$, and $>350,000$ people die from hepatitis-C-related liver diseases every year [2]. The standard treatment widely used for chronic hepatitis $\mathrm{C}(\mathrm{CHC})$ is a combination of peginterferon and ribavirin

\footnotetext{
*Correspondence: junqiniu@aliyun.com; yanzhang2006@sjtu.edu.cn ${ }^{\dagger}$ Equal contributors

${ }^{2}$ Department of Hepatology, First Hospital, Jilin University, Changchun 130021, China

${ }^{1}$ Ministry of Education Key Laboratory of Systems Biomedicine, Shanghai Center for Systems Biomedicine, Shanghai Jiao Tong University, 800 Dong Chuan Road, Minhang, Shanghai 200240, China

Full list of author information is available at the end of the article
}

$[3,4]$. The indication of successful therapy is the attainment of sustained virological response (SVR), which is defined as undetectable serum HCV RNA 24 weeks after treatment cessation [5]. With the current standard treatment, patients with chronic HCV infection show an SVR rate of $\sim 55 \%[6,7]$. This means that there is a large population of patients with treatment outcomes of no response, virological breakthrough, or relapse. Early prediction of the outcome during or after treatment is expected to provide additional information for individualizing treatment, and thus improves the cure rates for patients with chronic HCV infection.

One of the most important histological outcomes of interferon (IFN) therapy is the change in degree of fibrosis. Many studies have clearly shown that IFN therapy results in significant regression of fibrosis in patients who

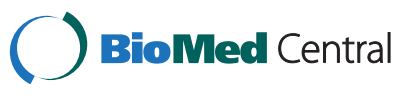

(C) 2014 Zou et al.; licensee BioMed Central. This is an Open Access article distributed under the terms of the Creative Commons Attribution License (http://creativecommons.org/licenses/by/4.0), which permits unrestricted use, distribution, and reproduction in any medium, provided the original work is properly credited. The Creative Commons Public Domain Dedication waiver (http://creativecommons.org/publicdomain/zero/1.0/) applies to the data made available in this article unless otherwise stated. 
attain SVR [8-10]. Thus, continuous monitoring of the degree of liver fibrosis should be beneficial for early estimation of the therapeutic efficacy and long-term followup of patients, which provides clues for the prognosis and management of CHC. It is evident that liver biopsy is considered as the gold standard for fibrosis staging [11]. This procedure has several disadvantages including invasiveness, potential complications, and sampling errors, which often limit its application, for example, frequent monitoring of the degree of fibrosis [12-14]. The development of noninvasive methods to complement liver biopsy is urgently needed. From this point of view, a variety of noninvasive methods has been developed, including physical techniques such as FibroScan [15] and serological tests such as FibroTest, Hepascore, enhanced liver fibrosis (ELF) index, platelets, APRI, and FIB-4 index [16-19]. FibroScan is recognized as a superior test for evaluation of fibrosis compared with biochemical markers [20]. It is restricted by the cost and the operator's experience and patient's body mass index (BMI) [21]. Many serological methods are also moderately useful for identifying significant fibrosis or cirrhosis in patients with chronic $\mathrm{HCV}$ infection. However, there are few serological tests reported to meet the above medical need.

Our previous study using glycomics technologies have developed and revealed a new fibrosis test named LecTHepa, which measures a glycobiomarker serum $\alpha 1$-acid glycoprotein (AGP) with fibrosis-related glyco-alterations performed well in estimating liver fibrosis [22]. It is correlated well with the fibrosis stage determined by liver biopsy [22-24] and FibroScan [25], either in a single-center $[22,23]$ or a multicenter study [24]. In the present study, continuous use of LecT-Hepa as an indicator of liver fibrosis during 48 weeks therapy with IFN and ribavirin led us to predict the outcome within the treatment period. We found that the change of LecT-Hepa just at the first 12 weeks of therapy could already distinguish $\mathrm{CHC}$ patients' attainment of SVR.

\section{Results}

Evaluation of the level of LecT-Hepa in serum and plasma specimens

LecT-Hepa has been shown as a reliable method for the evaluation of liver fibrosis [22,24,25]. However, previous studies were all conducted using serum specimens. To broaden the clinical application of LecT-Hepa, we compared the concentration of LecT-Hepa in serum and plasma prepared simultaneously from the same individuals. A total of 97 patients with confirmed $\mathrm{CHC}$ were included for this comparison (Table 1). As shown in Figure 1, we observed a significant linear correlation between the level of LecT-Hepa in serum and that in plasma $\left(R^{2}=0.6766, p<0.0001\right)$, with most of the patients ( 89 of 97 ) within the $95 \%$ confidence intervals of
Table 1 Clinical characteristics of the 142 CHC patients in this study

\begin{tabular}{|c|c|c|}
\hline & $\begin{array}{l}\text { CHC patients with } \\
\text { serum and plasma } \\
\text { specimens }(n=97)\end{array}$ & $\begin{array}{l}\text { CHC patients treated } \\
\text { with IFN and achieved } \\
\text { RVR }(n=45)\end{array}$ \\
\hline Age (year) & $52.30 \pm 8.20$ & $52.64 \pm 7.49$ \\
\hline Gender (male/female) & $55 / 42$ & $30 / 15$ \\
\hline TBIL $(\mu \mathrm{mol} / \mathrm{L})$ & $16.90 \pm 7.40$ & / \\
\hline DBIL $(\mu \mathrm{mol} / \mathrm{L})$ & $7.47 \pm 3.14$ & / \\
\hline ALP $(U / L)$ & $88.41 \pm 31.01$ & / \\
\hline GGT (U/L) & $79.79 \pm 91.21$ & / \\
\hline $\mathrm{ALT}(\mathrm{U} / \mathrm{L})$ & $57.88 \pm 50.87$ & $119.71 \pm 110.95$ \\
\hline AST (U/L) & $45.50 \pm 32.49$ & $87.70 \pm 82.70$ \\
\hline $\operatorname{PLT}\left(\times 10^{9} / \mathrm{L}\right)$ & $189.07 \pm 66.50$ & $166.60 \pm 83.20$ \\
\hline FibroScan & $9.44 \pm 10.22$ & $15.16 \pm 7.63$ \\
\hline \multirow[t]{2}{*}{ MAL/DSA } & Serum: $10.01 \pm 1.94$ & $9.29 \pm 2.39$ \\
\hline & Plasma: $10.35 \pm 2.30$ & \\
\hline \multirow[t]{2}{*}{ AOL/DSA } & Serum: $3.02 \pm 3.43$ & $6.34 \pm 7.33$ \\
\hline & Plasma: $3.57 \pm 4.78$ & \\
\hline
\end{tabular}

the correlation. In addition, according to the best linear curve with its correlation coefficient $(y=0.9653 x)$, the concentrations of LecT-Hepa in serum and plasma were almost the same. These data suggest that the serum and plasma specimens could both be used for clinical detection of LecT-Hepa.

\section{Baseline characteristics of $\mathbf{4 5}$ patients achieved rapid virological response (RVR)}

A total of $45 \mathrm{CHC}$ patients who had achieved RVR during IFN therapy and undergone 2 years of follow-up were used for the evaluation of the role of LecT-Hepa during hepatitis $\mathrm{C}$ treatment and follow-up. The mean age of the 45 patients was $52.64 \pm 7.49$ years, and $30(66.7 \%)$ of them were men (Table 1). To investigate the relation between the level of LecT-Hepa and fibrosis, these patients were divided into three groups based on the degrees of severity of liver fibrosis assessed by FibroScan. According to the study by Berzigotti et al. [26], 18 (40.0\%) patients with FibroScan value $<12 \mathrm{kPa}$ and 13 (28.9\%) with FibroScan value $\geq 18 \mathrm{kPa}$ were considered as non-cirrhosis (nonLC) and cirrhosis (LC), respectively. Other patients with FibroScan values of $12-18 \mathrm{kPa}$ were indeterminate. We also assessed the degree of fibrosis using color Doppler ultrasound. The results were highly consistent with the assessment by FibroScan (Table 2). In addition, the baseline characteristics of these patients are also summarized in Table 2. For the routine clinical indicators, platelet count (PLT) showed the most significant differences $(\mathrm{p}=0.004$ for non-LC $v s$. indeterminate, and $\mathrm{p}=0.001$ for non-LC $v s$. LC respectively, Student's $t$ test), while other indicators such as sex, alanine aminotransferase (ALT), and BMI did 


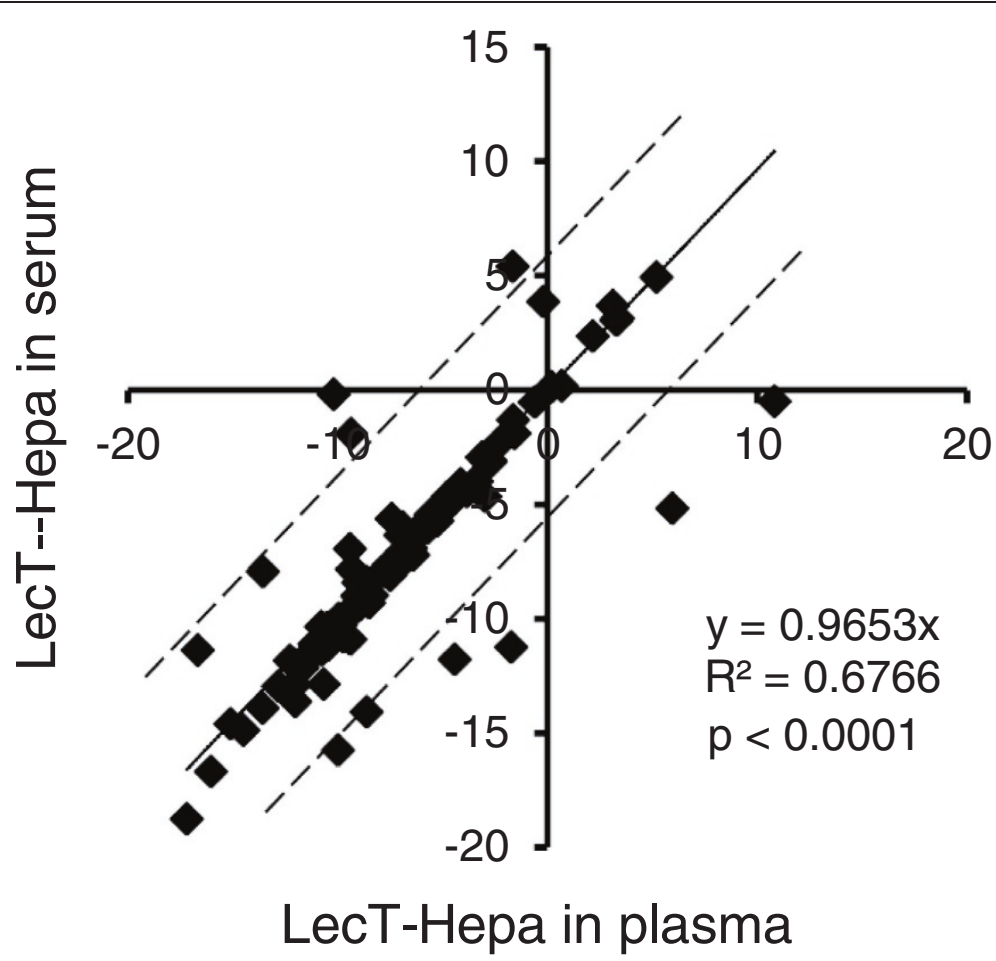

Figure 1 Correlation of concentrations of LecT-Hepa in serum and plasma specimens prepared simultaneously from the same individuals. The linear regression analysis was performed in 97 patients with confirmed CHC. The best-fit linear comparison with its correlation coefficient was calculated in Excel 2007 (Microsoft). The dotted line shows the 95\% confidence intervals of the correlation.

not differ significantly among these three groups. However, for the new indicator, both MAL/DSA and AOL/DSA always showed significant differences among the three groups. In addition, the univariate analysis revealed that the most significant differences were found between the
non-LC and LC groups, whereas the indeterminate and LC groups showed no difference. We observed a significant decrease in the level of MAL/DSA ( $p=2.68 \mathrm{E}-06$ vs. non$\mathrm{LC}$ ) and an increased level of AOL/DSA ( $\mathrm{p}=0.004$ vs. non-LC) in the LC group. These results suggest that the

Table 2 Baseline characteristics of the $45 \mathrm{HCV}$ patients in three different groups

\begin{tabular}{|c|c|c|c|c|c|c|}
\hline & \multirow{2}{*}{$\begin{array}{l}\text { Non-LC } \\
(n=18)\end{array}$} & \multirow{2}{*}{$\begin{array}{c}\text { Indeterminate } \\
\quad(n=14)\end{array}$} & \multirow{2}{*}{$\begin{array}{c}\text { LC } \\
(n=13)\end{array}$} & \multicolumn{3}{|c|}{ Significance } \\
\hline & & & & $\begin{array}{c}\text { Non-LC vs } \\
\text { indeterminate }\end{array}$ & $\begin{array}{c}\text { Indeterminate } \\
\text { vs LC }\end{array}$ & $\begin{array}{c}\text { Non-LC } \\
\text { vs LC }\end{array}$ \\
\hline Age (year) & $49.28 \pm 5.74$ & $55.00 \pm 8.64$ & $54.77 \pm 7.06$ & $p=0.032$ & $p=0.940$ & $p=0.024$ \\
\hline Gender (male/female) & $11 / 7$ & $12 / 2$ & $7 / 6$ & $p=0.235$ & $p=0.103$ & $p=0.727$ \\
\hline BMI & $23.15 \pm 3.01$ & $22.21 \pm 3.01$ & $23.62 \pm 3.19$ & $p=0.388$ & $p=0.248$ & $p=0.677$ \\
\hline AST (U/L) & $54.46 \pm 45.25$ & $110.05 \pm 100.31$ & $109.66 \pm 92.28$ & $p=0.044$ & $p=0.992$ & $p=0.035$ \\
\hline $\operatorname{ALT}(U / L)$ & $87.68 \pm 88.70$ & $137.44 \pm 111.08$ & $144.97 \pm 134.27$ & $p=-.169$ & $p=0.875$ & $p=0.163$ \\
\hline $\operatorname{PLT}\left(\times 10^{9} / \mathrm{L}\right)$ & $218.22 \pm 98.68$ & $140.64 \pm 46.25$ & $123.07 \pm 49.34$ & $p=0.011$ & $p=0.349$ & $p=0.003$ \\
\hline MAL/DSA & $11.02 \pm 1.44$ & $8.82 \pm 2.22$ & $7.41 \pm 2.03$ & $p=0.002$ & $p=0.100$ & $p=2.68 E-06$ \\
\hline AOL/DSA & $1.94 \pm 1.08$ & $6.42 \pm 4.14$ & $12.35 \pm 10.41$ & $p=0.001$ & $p=0.059$ & $p=0.004$ \\
\hline \multicolumn{7}{|c|}{ Color Doppler ultrasound assessment* } \\
\hline 1 & $87.5 \%(14 / 16)$ & $50.0 \%(6 / 12)$ & $0.0 \%(0 / 11)$ & $p=0.044$ & $p=0.014$ & $p=5.98 E-06$ \\
\hline 2 & $12.5 \%(2 / 16)$ & $16.7 \%(2 / 12)$ & $45.4 \%(5 / 11)$ & $p=1.000$ & $p=0.193$ & $p=0.084$ \\
\hline 3 & $0.0 \%(0 / 16)$ & $33.3 \%(4 / 12)$ & $36.4 \%(4 / 11)$ & $p=0.024$ & $p=1.000$ & $p=0.019$ \\
\hline 4 & $0.0 \%(0 / 16)$ & $0.0 \%(0 / 12)$ & $18.2 \%(2 / 11)$ & $p=1.000$ & $p=0.217$ & $p=0.157$ \\
\hline
\end{tabular}

*2 patients in each group were not measured by color Doppler ultrasound. 
new indicator LecT-Hepa may be superior to the routine clinical indicators for the evaluation of fibrosis in this cohort.

\section{Evaluation of LecT-Hepa, FIB-4, and APRI for estimating progression of liver fibrosis during IFN treatment of HCV-infected patients}

In $\mathrm{HCV}$-infected patients, evaluation of the progression of fibrosis is an important indicator of antiviral therapy [17]. However, only a few serum markers have been reported for predicting fibrosis progression and regression during treatment. Here, we investigated the relation between LecT-Hepa and fibrosis progression. First, we performed a correlation analysis of LecT-Hepa against the fibrosis levels measured by FibroScan at different times during treatment. As shown in Figure 2, we observed a significant linear correlation between the level of LecT-Hepa and FibroScan before $\left(0\right.$ weeks $R^{2}=0.6790$, $\mathrm{p}<0.0001)$ and after (24 weeks, $\mathrm{R}^{2}=0.6387, \mathrm{p}=0.0077$ and 48 weeks, $\left.\mathrm{R}^{2}=0.7311, \mathrm{p}=0.0006\right)$ treatment. This suggests that change in LecT-Hepa reflects a change in FibroScan during IFN treatment. Then, we performed a trend analysis of LecT-Hepa, FIB-4, and APRI during 48 weeks of IFN therapy. As shown in Figure 3A, all $\mathrm{CHC}$ patients in the non-LC group had LecT-Hepa values $<0$, while the mean level of LecT-Hepa in patients with LC was $>0$. A gradually increasing trend of LecTHepa from the non-LC to the LC group was observed. The difference in LecT-Hepa between the three groups was significant at different times during treatment ( $\mathrm{p}<0.0001$ for $0,4,12$ and 24 weeks). The level of FIB-4 was also higher in the LC than in the non-LC group, and the mean level of FIB- 4 in the LC group was higher than the reference cutoff value of 3.45 for cirrhosis [27]. However, this trend was not obvious and regular in all patients. In contrast, APRI showed lesser changes between the non-LC and LC groups. These results indicated that LecT-Hepa was effective for evaluation of the progression of fibrosis, at least in this cohort.

To investigate the change in LecT-Hepa during the 48-week course of IFN therapy in detail, we analyzed the levels of LecT-Hepa, FIB-4, and APRI at 0, 4, 12, 24, and
48 weeks of therapy in $45 \mathrm{CHC}$ patients (Additional file 1: Figure S1 and Table 3). The mean level of LecT-Hepa was increased from -4.69 to -3.25 in the LC group $(p=0.076$, paired $t$ test) during the early phase of therapy (0-4 weeks), followed by a small but meaningful reduction after viral elimination (from 4 to 12,24 , and 48 weeks) (the mean value from -3.25 to $-3.24,-4.19$ and -7.31 , $\mathrm{p}=0.029$ from 4 to 24 weeks, $\mathrm{p}=0.026$ from 12 to 24 weeks). For the other two indices, APRI showed a dramatic decrease during the early stage of IFN treatment (0-4 weeks) ( $\mathrm{p}=0.0009$, paired $t$ test), followed by a more stable trend (mean value from 0.81 to $0.83,0.78$ and 0.76 , $\mathrm{p}=0.275$, one-way ANOVA), whereas FIB-4 showed no clear regular changes during IFN treatment. Combined with the significant correlation of LecT-Hepa and FibroScan, we suggest that the change in LecT-Hepa is superior to FIB-4 and APRI for describing the changes in fibrosis during IFN treatment in this cohort.

\section{Evaluation of the role of LecT-Hepa in prognosis of patients with HCV}

To evaluate the relationship between changes in LecTHepa and prognosis of $\mathrm{CHC}$ patients, we compared the levels of LecT-Hepa, FIB-4, and APRI in 45 patients who attained RVR with different treatment outcomes (SVR or non-SVR). Patients who attained RVR had undetectable HCV RNA after 4 weeks of therapy. We compared the clinical characteristics including the serum index between SVR and non-SVR groups (Table 4). For the serum indicators LecT-Hepa, FIB-4, and APRI, we calculated the Rvalue given as the sum of the changes from 4 to 12 weeks ( $\mathrm{R}=12-4$ weeks) during IFN treatment, which showed the variation after viral elimination and reflected the early outcome of treatment. Besides the effect of serum HCV RNA level on treatment outcome, it is worth noting that at 12 weeks of therapy, only $\mathrm{R}$ value of LecT-Hepa showed a significant difference ( $\mathrm{p}=0.0031$, Mann-Whitney $U$ test) between SVR and non-SVR groups, while those of the other two indicators were not ( $p=0.5545$ for FIB- 4 and $\mathrm{p}=0.7626$ for APRI) (Figure 4A). Those results suggest that the change in LecT-Hepa at the first 12 weeks of therapy was more sensitive in predicting the treatment
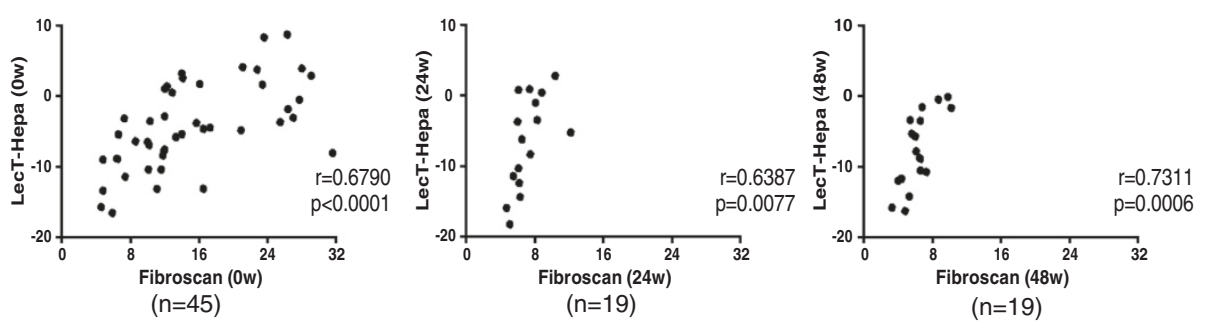

Figure 2 Correlation of concentrations of LecT-Hepa and FibroScan values at baseline ( 0 w), 24 weeks (24 w) and 48 weeks (48 w) of the treatment process. 


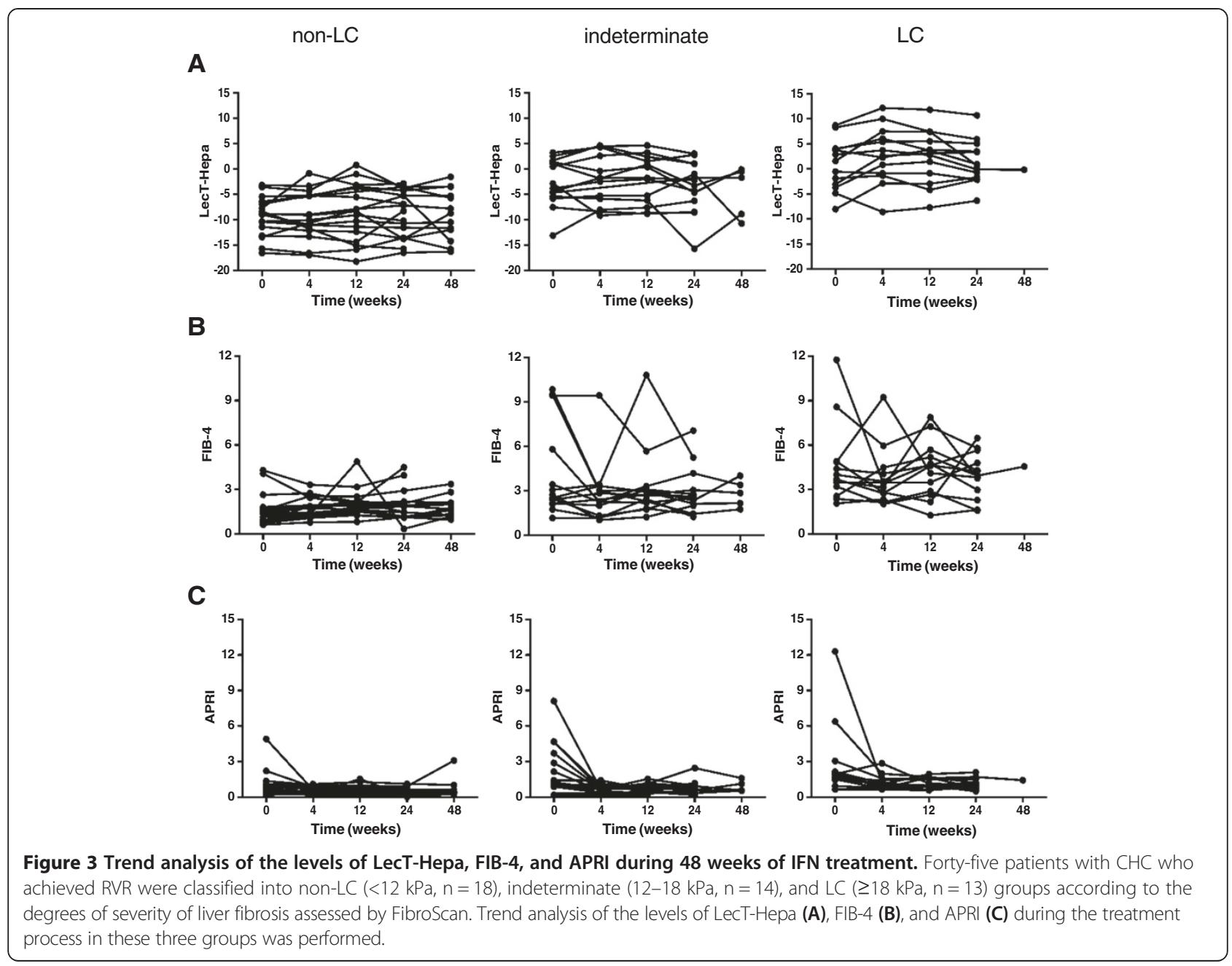

Table 3 Levels of LecT-Hepa, FIB-4 and APRI in45 CHC patients during 48 weeks course of IFN therapy

\begin{tabular}{|c|c|c|c|c|c|}
\hline Weeks & 0 & 4 & 12 & 24 & 48 \\
\hline \multicolumn{6}{|l|}{ LecT-Hepa } \\
\hline Non-LC $(n=18)$ & $-9.22 \pm 3.77$ & $-9.09 \pm 4.66$ & $-8.72 \pm 5.66$ & $-8.71 \pm 4.69$ & $-8.98 \pm 4.88$ \\
\hline Indeterminate $(n=14)$ & $-2.10 \pm 4.63$ & $-2.14 \pm 5.02$ & $-2.12 \pm 4.72$ & $-3.47 \pm 5.13$ & $-4.40 \pm 5.01$ \\
\hline $\operatorname{LC}(n=13)$ & $0.83 \pm 5.06$ & $2.82 \pm 5.60$ & $2.38 \pm 5.32$ & $1.29 \pm 4.39$ & -0.17 \\
\hline Total $(n=45)$ & $-4.69 \pm 6.11$ & $-3.25 \pm 7.01$ & $-3.24 \pm 6.97$ & $-4.19 \pm 6.21$ & $-7.31 \pm 5.35$ \\
\hline \multicolumn{6}{|l|}{ F|B-4 } \\
\hline Non-LC $(n=18)$ & $1.63 \pm 1.06$ & $1.72 \pm 0.70$ & $2.03 \pm 0.94$ & $1.87 \pm 1.03$ & $1.81 \pm 0.68$ \\
\hline Indeterminate $(n=14)$ & $4.17 \pm 3.11$ & $2.79 \pm 2.17$ & $3.33 \pm 2.49$ & $2.91 \pm 1.61$ & $2.84 \pm 0.91$ \\
\hline$L C(n=13)$ & $4.57 \pm 2.71$ & $3.80 \pm 1.95$ & $4.35 \pm 1.91$ & $3.94 \pm 1.53$ & 4.55 \\
\hline Total $(n=45)$ & $3.27 \pm 2.68$ & $2.70 \pm 1.85$ & $3.15 \pm 2.04$ & $2.79 \pm 1.60$ & $2.22 \pm 1.01$ \\
\hline \multicolumn{6}{|l|}{ APRI } \\
\hline Non-LC $(n=18)$ & $0.96 \pm 1.11$ & $0.54 \pm 0.27$ & $0.58 \pm 0.36$ & $0.51 \pm 0.29$ & $0.66 \pm 0.76$ \\
\hline Indeterminate $(n=14)$ & $2.44 \pm 2.18$ & $0.70 \pm 0.32$ & $0.76 \pm 0.37$ & $0.82 \pm 0.56$ & $0.89 \pm 0.48$ \\
\hline$L C(n=13)$ & $2.92 \pm 3.15$ & $1.26 \pm 0.61$ & $1.19 \pm 0.45$ & $1.10 \pm 0.46$ & 1.44 \\
\hline Total $(n=45)$ & $1.98 \pm 2.31$ & $0.81 \pm 0.52$ & $0.83 \pm 0.46$ & $0.78 \pm 0.49$ & $0.76 \pm 0.69$ \\
\hline
\end{tabular}


Table 4 Clinical characteristics of the SVR and non-SVR patients

\begin{tabular}{|c|c|c|c|}
\hline & $\begin{array}{l}\text { SVR patients } \\
(n=23)^{1}\end{array}$ & $\begin{array}{l}\text { Non-SVR patients } \\
(\mathrm{n}=18)^{1}\end{array}$ & Significance \\
\hline Age (year) & $52.13 \pm 7.86$ & $53.78 \pm 7.20$ & $p=0.4530$ \\
\hline Gender & & & $p=0.7020$ \\
\hline Male & 14 & 12 & \\
\hline Female & 9 & 6 & \\
\hline BMI & $23.22 \pm 2.98$ & $22.27 \pm 2.95$ & $p=0.3510$ \\
\hline $\operatorname{ALT}(\mathrm{U} / \mathrm{L})^{2}$ & $156.59 \pm 134.65$ & $80.74 \pm 67.90$ & $p=0.0659$ \\
\hline $\mathrm{AST}(\mathrm{U} / \mathrm{L})^{2}$ & $104.59 \pm 97.74$ & $65.27 \pm 59.63$ & $p=0.1180$ \\
\hline $\operatorname{PLT}\left(\times 10^{9} / L\right)^{2}$ & $138.30 \pm 57.34$ & $205.94 \pm 102.76$ & $p=0.0551$ \\
\hline AFP $^{2}$ & $2.99 \pm 1.23$ & $4.68 \pm 2.96$ & $p=0.0841$ \\
\hline $\begin{array}{l}\text { HCV RNA } \\
\left(\times 10^{6} \mathrm{eq} / \mathrm{mL}\right)^{2}\end{array}$ & $1.15 \pm 1.60$ & $8.78 \pm 9.92$ & $p=0.0051$ \\
\hline FibroScan ${ }^{2}$ & $17.16 \pm 7.98$ & $13.56 \pm 7.15$ & $p=0.1412$ \\
\hline $\begin{array}{l}\text { Liver fibrosis } \\
\text { assessed } \\
\text { by FibroScan }\end{array}$ & & & $p=0.2170$ \\
\hline Non-LC & 7 & 9 & \\
\hline Indeterminate & 6 & 6 & \\
\hline LC & 10 & 3 & \\
\hline R of LecT-Hepa & $-0.60 \pm 1.48$ & $0.79 \pm 1.54$ & $p=0.0031$ \\
\hline R of FIB4 & $0.38 \pm 2.00$ & $0.62 \pm 1.76$ & $p=0.5545$ \\
\hline $\mathrm{R}$ of APRI & $0.01 \pm 0.60$ & $0.04 \pm 0.28$ & $p=0.7626$ \\
\hline
\end{tabular}

${ }^{1)} 4$ of 45 patients were lost to follow-up.

${ }^{2)}$ Clinical information was the baseline ( 0 weeks) information.

outcome than FIB-4 and APRI were. In addition, from this preliminary result, we found that $\mathrm{R}$ value of LecTHepa were higher in patients who have not attained SVR.

Furthermore, to evaluate the overall diagnostic performances and attempt to establish clinically useful cut-off levels of these serum indices, we constructed receiveroperating characteristic (ROC) curves for R-values of LecT-Hepa, FIB-4, and APRI. As shown in Figure 4B, the area under the curve (AUC) $(95 \% \mathrm{CI})$ of LecT-Hepa for distinguishing between SVR and non-SVR patients $(0.773,0.615-0.889)$ was superior to FIB-4 $(0.556,0.392-$ 0.720 ) and APRI (0.471, 0.314-0.633), and the difference were significant between LecT-Hepa and the other two indicators $(\mathrm{p}=0.043$ vs. FIB- 4 and $\mathrm{p}=0.011$ vs. APRI). Based on Youden's index from the ROC curve, the optimal cut-off value of LecT-Hepa was -0.0934 , with sensitivity of $83.33 \%$, specificity of $60.87 \%$, positive predictive value (PPV) of $62.5 \%$, and negative predictive value (NPV) of $82.4 \%$. These results implied that the change in the serum level of glycoprotein LecT-Hepa could predict the antiviral treatment response more quickly than FIB-4 and APRI, even at the first 12 weeks of therapy, which may provide more precise information for treatment protocols of $\mathrm{CHC}$.

\section{Discussion}

For patients with $\mathrm{CHC}$, the traditional therapy is a combination of IFN and ribavirin. Recently, with the development of many other drugs targeting viral or host factors, and the approval of two direct-acting antiviral agents $[28,29]$, the question of who should be treated and with what regimen has become increasingly complex to address and needs more careful consideration [3]. Liver biopsy is considered as the gold standard for fibrosis staging. However, it cannot be used for continuously monitoring the progression of hepatitis because of its invasiveness and lack of accuracy. Thus, developing noninvasive tests like serum indictors that could continuously monitor the histological progression of hepatitis during therapy is beneficial for providing information for physicians and optimization of treatment. At present, a few biomarkers have been reported to predict the response to IFN-based regimens before the start of antiviral therapy [30-32]. For example, a recent study has suggested that patients with a favorable interleukin-28 (IL28B) genotype can receive peginterferon and ribavirin first, with the approved triple therapy subsequently if the initial treatment fails [33]. In addition, the pretreatment interferongamma-inducible protein-10 (IP-10) levels in plasma can predict RVR and SVR in patients infected with $\mathrm{HCV}$ genotype 1 , and thus may be helpful in decision making regarding pharmaceutical intervention [34]. However, it should be stressed that there are few biomarkers that can monitor the progression of hepatitis during therapy. Thus, in this study, we focused on the potential predictive value of serum LecT-Hepa level during treatment with IFN and ribavirin. We analyzed the clinical information, including serum levels of LecT-Hepa, FIB4, and APRI. We clearly showed changes in serum level of LecT-Hepa during IFN treatment. We are particularly interested in the small reduction in LecT-Hepa after viral elimination (from 4 to 12 and 24 weeks) because at that time fibrosis began to ease $[35,36]$. Based on the significant correlation of LecTHepa and FibroScan, we speculate that the change in LecT-Hepa may reflect the changes in fibrosis during IFN treatment (Figures 2 and 3). We only used RVR patients in this study; all of whom had a $\geq 2 \log 10$ decrease in HCV RNA level by 4 weeks of therapy. SVR patients maintained a low or undetectable HCV RNA level during and after therapy. However, non-SVR patients showed virological breakthrough or relapse during or after therapy (Additional file 2: Figure S2). Serum levels of ALT and AST for SVR and non-SVR patients showed a similar tendency, with a dramatic decrease at $0-4$ weeks, followed by a more stable trend. The HCV RNA quantitation became to decrease and the liver function returned to normal is the clinical indicators to determine the treatment outcome. During this process, LecT-Hepa showed a decrease just after viral elimination (4-12 weeks) for SVR patients 

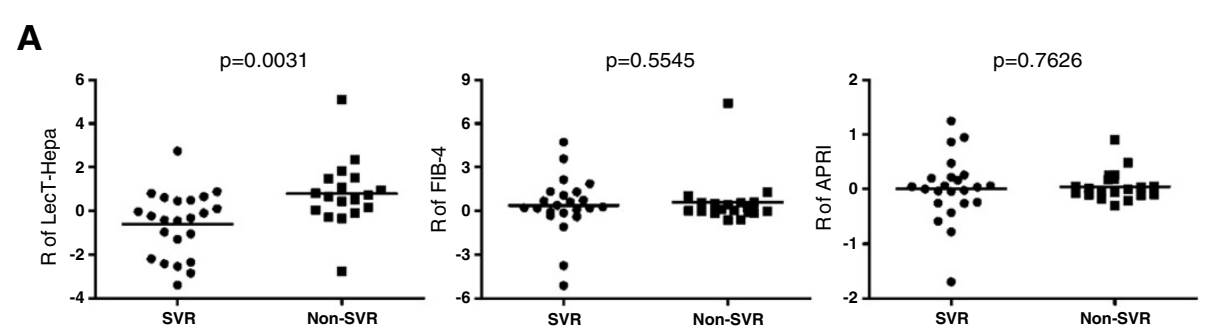

\section{B}

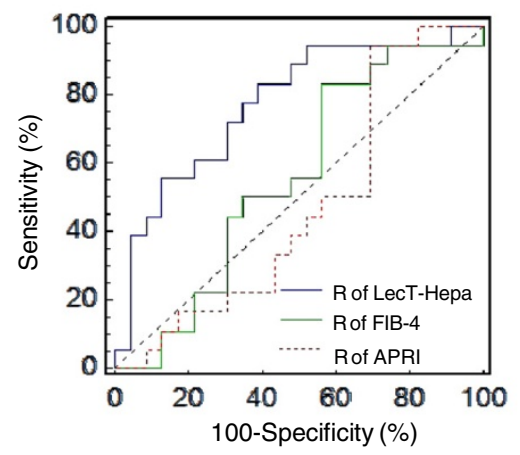

\begin{tabular}{|c|c|c|c|}
\hline & $\begin{array}{c}\text { R of } \\
\text { LecT-Hepa }\end{array}$ & $\begin{array}{c}\text { R of } \\
\text { FlB-4 }\end{array}$ & $\begin{array}{c}\text { R of } \\
\text { APRI }\end{array}$ \\
\hline Cut-off value & -0.0934 & 0.606 & -0.2395 \\
\hline $\begin{array}{c}\text { AUC } \\
(95 \% \text { Cl) }\end{array}$ & $\begin{array}{c}0.773 \\
(0.615-0.889)\end{array}$ & $\begin{array}{c}0.556 \\
(0.392-0.720)\end{array}$ & $\begin{array}{c}0.471 \\
(0.314-0.633)\end{array}$ \\
\hline $\mathrm{Se}(\%)$ & 83.33 & 83.33 & 94.44 \\
\hline $\mathrm{Sp}(\%)$ & 60.87 & 43.48 & 30.43 \\
\hline $\mathrm{PPV}(\%)$ & 62.5 & 53.6 & 51.5 \\
\hline $\mathrm{NPV}(\%)$ & 82.4 & 76.9 & 87.5 \\
\hline
\end{tabular}

Figure 4 Evaluation of roles of R-value LecT-Hepa, FIB-4, and APRI in predicting treatment outcome of HCV. (A) Serum levels of LecT-Hepa, FIB-4, and APRI from 4 to 12 weeks ( $R=12-4$ weeks) were calculated in SVR $(n=23)$ and non-SVR $(n=18)$ patients who achieved RVR during the 48-week course of IFN therapy and underwent a 2-year period of follow-up (4 of the 45 patients were lost to follow-up). Mean values are indicated by a horizontal line and $p$ values were calculated by the Mann-Whitney $U$ test. (B) ROC curves of LecT-Hepa, FIB-4, and APRI for distinguishing patients with SVR from non-SVR. The cut-off values were based on the Youden's index from the ROC curve. Se, sensitivity; Sp, specificity.

while it showed a late decrease after viral elimination (12-24 weeks) for non-SVR patients (Additional file 2: Figure S2). Our data also showed that the change in LecTHepa was well correlated with the treatment outcome of $\mathrm{CHC}(\mathrm{p}=0.0031)$. If patients had an increased $\mathrm{R}$ value in LecT-Hepa ( $\mathrm{R}=12-4$ weeks), they were more likely to experience relapse and become non-SVR (Figure 4).

Currently, the mechanism of relapse is not fully understood but several factors have been reported as risk factors for relapse and response [37], such as viral genotype 1 [38], high viral load [39], metabolic factors [40], shorter treatment with inadequate doses of ribavirin, and the degree of liver fibrosis and cirrhosis [41]. Previous reports have suggested that the index LecT-Hepa is one of the best candidates for glyco-indicators in liver fibrosis. LecT-Hepa count is based on the glyco-alternation in serum AGP. AGP is mainly synthesized in the liver and its glycosylation has a profound effect on collagen fibril formation [42,43]. Goodman and Marcellin et al. have reported that the degree of liver fibrosis is characterized by a linear increase in fibrillar collagen, which was more resistant to enzymatic degradation in their studies [44,45]. Thus, we speculate that LecT-Hepa level shows a linear correlation with the degree of fibrosis. Now, we understand the relation between LecT-Hepa level and treatment outcome. If the $\mathrm{R}$ value (12-4 weeks) is larger, it indicates that the degree of fibrosis at 12 weeks is more severe than at 4 weeks. That means that after treatment, liver fibrosis is not relieved and may become more severe. In other words, the treatment is not effective in these patients, and they will likely not attain an SVR. In addition, because the coagulation process did not affect glycosylation of AGP, we found that the level of LecT-Hepa showed no difference in serum and plasma. We also compared the LecT-Hepa levels in patients with HCV genotype $1 \mathrm{a}$ and $2 \mathrm{~b}$ (Additional file 3: Figure S3). Those results showed that 
the level of LecT-Hepa was not affected by sample type or $\mathrm{HCV}$ genotype, and the change in LecT-Hepa level indeed reflected the therapeutic efficacy.

To the best of our knowledge, this is the first study to investigate the noninvasive serum glyco-marker as a predictive factor for prognosis of $\mathrm{CHC}$ patients undergoing treatment. The prognostic value of serum LecT-Hepa level is superior to that of other biochemical markers such as FIB-4 and APRI just at the first 12 weeks of therapy. In addition, because the level of LecT-Hepa is positively correlated with the degree of fibrosis, it may be used for liver function monitoring at optimal intervals and for the prediction of the treatment outcome of new antifibrotic drugs.

\section{Conclusions}

In summary, this study was a trial for the estimation of therapeutic efficacy in patients with $\mathrm{CHC}$ using serum glycoproteins. It is an extension of previous study which has found LecT-Hepa as a good predictor of fibrosis using glycomics technologies. Our study revealed that the change in serum level of LecT-Hepa after viral elimination may serve as an early predictor of antiviral treatment response in $\mathrm{CHC}$ patients treated with IFN and ribavirin, and may provide additional information for individualizing treatment. This study provides evidence for the clinical value of serum glycomics and gives a new perspective that the serum glyco-marker could be used as a joint indicator target of disease.

\section{Materials and methods \\ Patients}

A total of 142 patients with a positive anti-HCV antibody and HCV viral load were enrolled from the Department of Hepatology, First Hospital of Jilin University. Patients were enrolled after August 2010 and followed up for at least 48 weeks. Inclusion criteria were (1): diagnosis with $\mathrm{CHC}$; and (2) HCV RNA was positive as determined by the COBAS TaqMan HCV test (Roche Diagnostics, Branchburg, NJ, USA). Exclusion criteria were: (1) coinfection with another hepatitis virus or HIV; (2) excessive alcohol intake; (3) hepatocellular carcinoma or its history; and (4) decompensated liver cirrhosis.

This retrospective cohort study was divided into two parts: One part contained 97 patients with sera and plasma collected simultaneously. The other part included 213 serum specimens from the remaining 45 patients who received 48 weeks treatment with IFN and ribavirin, and were followed up for 96 weeks. All of the 45 patients achieved an RVR with $\geq 2 \log _{10}$ decrease in HCV RNA level by 4 weeks of therapy. This study was in accordance with the ethical guidelines of the 1975 Declaration of Helsinki and was approved by the Ethical Committee of the First Hospital, Jilin University. Each participant gave written informed consent.

\section{Detection and quantification of HCV RNA}

The concentration of HCV RNA in serum was determined by reverse transcriptase polymerase chain reaction using the COBAS TaqMan HCV assay (Roche Diagnostics). Serum was collected at different time points during therapy and follow-up $(0,4,12,24,48,60,72,96$, and 144 weeks). According to the viral kinetic response and treatment outcome, 45 patients were judged as SVR with undetectable HCV RNA 24 weeks after therapy was complete, or as non-SVR.

\section{Clinical and biological data}

The basic anthropometric parameters, such as age and sex of the patients were recorded. Serum and plasma samples were collected and stored at $-80^{\circ} \mathrm{C}$ until analysis. The serum biochemical parameters, including concentrations of total bilirubin (TBIL), direct bilirubin (DBIL), alkaline phosphatase (ALP), $\gamma$-glutamyltransferase (GGT), ALT, aspartate aminotransferase (AST) and PLT were assessed by the medical laboratory of the First Hospital of Jilin University. The APRI and FIB-4 index were calculated according to published formulas $[46,47]$.

\section{Liver stiffness measurement}

Liver stiffness was measured by transient elastography using FibroScan (EchoSens, Paris, France). The measurement depth was between 25 and $65 \mathrm{~mm}$. For each patient, 10 validated measurements were performed. The success rate was calculated as the number of validated measurements divided by the total number of measurements. The results were expressed in kilopascals. The median value was considered representative of the elastic modulus of the liver. Only procedures with 10 validated measurements and a success rate of at least $60 \%$ were considered reliable.

\section{Automatic acquisition of quantitative glyco-alteration of AGP (LecT-Hepa)}

The detailed procedure for LecT-Hepa has been described previously $[22,25]$. Each individual serum or plasma sample $(5 \mu \mathrm{L})$ was diluted and heated at $95^{\circ} \mathrm{C}$ for $20 \mathrm{~min}$ before enrichment of AGP. The AGP in the sample was enriched by immunoprecipitation with a biotinylated antiAGP antibody using an automated protein purification system (ED-01; GP BioSciences, Tokyo, Japan). Finally, fibrosis-specific glyco-alteration of the enriched AGPs was determined by lectin-antibody sandwich immunoassays with a combination of three lectins (Datura stramonium agglutinin (DSA), Maackia amurensis leukoagglutinin (MAL), and Aspergillus oryzae lectin (AOL)) [23] using an automated chemiluminescence enzyme immunoassay system (HISCL-2000i; Sysmex, Kobe, Japan). The criterion formula of LecT-Hepa was as follows [22]: LecT-Hepa = $\log _{10}[\mathrm{AOL} / \mathrm{DSA}] \times 8.6-[\mathrm{MAL} / \mathrm{DSA}]$. 


\section{Statistical analysis}

Statistical calculations were conducted with Microsoft Office Excel and SPSS version 16.0 statistical package (SPSS, Chicago, IL, USA). Categorical data were analyzed using $X^{2}$ test and continuous variables were compared with the Student's $t$ test or Mann-Whitney $U$ test. In addition to assessing the predictive ability of various markers to differentiate SVR from non-SVR patients, ROC curve analysis was performed. Diagnostic accuracy was expressed as the diagnostic specificity, sensitivity, PPV, NPV, and AUC. The cutoff values were obtained from Youden's index [48]. A p value $<0.05$ in all cases was considered statistically significant.

\section{Additional files}

Additional file 1: Figure S1. Trend analysis of the levels of LecT-Hepa, FIB-4, and APRI during 48 weeks of IFN treatment in $45 \mathrm{CHC}$ patients.

Additional file 2: Figure S2. Clinical information for SVR and non-SVR patients at 0-48 weeks.

Additional file 3: Figure S3. Relation of the levels of LecT-Hepa, FIB-4, and APRI with HCV genotype. We compared the levels of LecT-Hepa, FIB-4, and APRI during 48 weeks of IFN therapy in patients with different HCV genotype (dot: HCV genotype 1b; circle: HCV genotype 2a).

\begin{abstract}
Abbreviations
CHC: Chronic hepatitis C; HCV: Hepatitis C virus; IFN: Interferon; SVR: Sustained virological response; RVR: Rapid virological response; ELF: Enhanced liver fibrosis; AGP: a1-acid glycoprotein; DSA: Datura stramonium agglutinin; MAL: Maackia amurensis leukoagglutinin; AOL: Aspergillus oryzae lectin; APRI: Aspartate aminotransferase-to-platelet ratio index; TBIL: Total bilirubin; DBIL: Direct bilirubin; ALP: Alkaline phosphatase; GGT: $\gamma$-glutamyltransferase; ALT: Alanine aminotransferase; AST: Aspartate aminotransferase; PLT: Platelet count; BMI: Body mass index; ROC: Receiver-operating characteristic; AUC: Area under the curve; PPV: Positive predictive value; NPV: Negative predictive value; IP-10: Interferon-gamma-inducible protein-10; IL28B: Interleukin-28B.
\end{abstract}

\section{Competing interests}

The authors declare that they have no competing interests.

\begin{abstract}
Authors' contributions
ZX (Xia Zou) participated in the organization of the clinical information, and performed the statistical analysis and drafted the manuscript. CX participated in the sample collection and discussion of clinical issues. PY and SH participated in the collection of serum and plasma specimens. DD and MA carried out the detection of LecT-Hepa in the clinical specimens. LW participated in the organization and transport of the clinical samples. KA participated in the detection of LecT-Hepa and discussion of this study. ZX (Xinxin Zhang) participated in the discussion of clinical issues. $\mathrm{NH}$ conceived of the study, and participated in its design and coordination. NJ participated in its design and coordination and discussion of clinical issues. ZY participated in its design and coordination and helped to draft the manuscript. All authors read and approved the final manuscript.
\end{abstract}

\section{Acknowledgements}

This work was financially supported by the grants from the International Science \& Technology Cooperation Program of China (2012DFG32190), the Shanghai International Science \& Technology Cooperation Program of China (12410707200), the National Basic Research Program of China (2012CB822103) and the National High Technology Research and Development Program of China (2012AA020203)

\section{Author details}

${ }^{1}$ Ministry of Education Key Laboratory of Systems Biomedicine, Shanghai Center for Systems Biomedicine, Shanghai Jiao Tong University, 800 Dong Chuan Road, Minhang, Shanghai 200240, China. 'Department of Hepatology, First Hospital, Jilin University, Changchun 130021, China. ${ }^{3}$ Research Center for Medical Glycoscience (RCMG), National Institute of Advanced Industrial Science and Technology (AIST), 1-1-1 Umezono, Tsukuba, Ibaraki 305-8568, Japan. ${ }^{4}$ SCSB (China) - AIST (Japan) Joint Medical Glycomics Laboratory, 800 Dong Chuan Road, Minhang, Shanghai 200240, China. ${ }^{5}$ Department of Infectious Diseases, Ruijin Hospital, Shanghai Jiao Tong University School of Medicine, 197, Ruijin Er Road, Shanghai 200025, China. 'Department of Surgery, Ruijin Hospital, Shanghai Jiao Tong University School of Medicine, 197, Ruijin Er Road, Shanghai 200025, China.

Received: 25 September 2014 Accepted: 25 November 2014 Published: 11 December 2014

\section{References}

1. Brody H: Hepatitis C. Nature 2011, 474:S1.

2. World Health Organization HepatitisC Fact Sheet. 2013. http://www.who.int/ mediacentre/factsheets/fs164/en/. Accessed 5 December 2013.

3. Liang TJ, Ghany MG: Current and future therapies for hepatitis C virus infection. N Engl J Med 2013, 368:1907-1917.

4. Casey LC, Lee WM: Hepatitis C virus therapy update 2013. Curr Opin Gastroenterol 2013, 29:243-249.

5. Pearlman BL, Traub N: Sustained virologic response to antiviral therapy for chronic hepatitis $C$ virus infection: a cure and so much more. Clin Infect Dis 2011, 52:889-900.

6. Manns MP, McHutchison JG, Gordon SC, Rustgi VK, Shiffman M, Reindollar R, Goodman ZD, Koury K, Ling M, Albrecht JK: Peginterferon alfa-2b plus ribavirin compared with interferon alfa-2b plus ribavirin for initial treatment of chronic hepatitis C: a randomised trial. Lancet 2001, 358:958-965.

7. Fried MW, Shiffman ML, Reddy KR, Smith C, Marinos G, Goncales FL Jr, Haussinger D, Diago M, Carosi G, Dhumeaux D, Craxi A, Lin A, Hoffman J, Yu J: Peginterferon alfa-2a plus ribavirin for chronic hepatitis $C$ virus infection. N Engl J Med 2002, 347:975-982.

8. Shiratori Y, Imazeki F, Moriyama M, Yano M, Arakawa Y, Yokosuka O, Kuroki T, Nishiguchi S, Sata M, Yamada G, Fujiyama S, Yoshida H, Omata M: Histologic improvement of fibrosis in patients with hepatitis $C$ who have sustained response to interferon therapy. Ann Intern Med 2000, 132:517-524.

9. Sobesky R, Mathurin P, Charlotte F, Moussalli J, Olivi M, Vidaud M, Ratziu V, Opolon P, Poynard T: Modeling the impact of interferon alfa treatment on liver fibrosis progression in chronic hepatitis C: a dynamic view. The Multivirc Group. Gastroenterology 1999, 116:378-386.

10. Toccaceli F, Laghi V, Capurso L, Koch M, Sereno S, Scuderi M, Italian Hepanet G: Long-term liver histology improvement in patients with chronic hepatitis $C$ and sustained response to interferon. J Viral Hepat 2003, 10:126-133.

11. Ghany MG, Strader DB, Thomas DL, Seeff LB, American Association for the Study of Liver D: Diagnosis, management, and treatment of hepatitis C: an update. Hepatology 2009, 49:1335-1374.

12. Cadranel JF, Rufat $P$, Degos F: Practices of liver biopsy in France: results of a prospective nationwide survey. For the Group of Epidemiology of the French Association for the Study of the Liver (AFEF). Hepatology 2000, 32:477-481.

13. Bravo AA, Sheth SG, Chopra S: Liver biopsy. N Engl J Med 2001, 344:495-500.

14. Regev A, Berho M, Jeffers LJ, Milikowski C, Molina EG, Pyrsopoulos NT, Feng ZZ, Reddy KR, Schiff ER: Sampling error and intraobserver variation in liver biopsy in patients with chronic HCV infection. Am J Gastroenterol 2002, 97:2614-2618.

15. D'Ambrosio R, Aghemo A, Fraquelli M, Rumi MG, Donato MF, Paradis V, Bedossa P, Colombo M: The diagnostic accuracy of Fibroscan for cirrhosis is influenced by liver morphometry in HCV patients with a sustained virological response. J Hepatol 2013, 59:251-256.

16. Chou R, Wasson N: Blood tests to diagnose fibrosis or cirrhosis in patients with chronic hepatitis C virus infection. Ann Intern Med 2013, 159:372. 
17. Castera L: Non-invasive assessment of liver fibrosis in chronic hepatitis C. Hepatol Int 2011, 5:625-634.

18. Rosenberg WM, Voelker M, Thiel R, Becka M, Burt A, Schuppan D, Hubscher S, Roskams T, Pinzani M, Arthur MJ, European Liver Fibrosis G: Serum markers detect the presence of liver fibrosis: a cohort study. Gastroenterology 2004, 127:1704-1713.

19. Cales P, Oberti F, Michalak S, Hubert-Fouchard I, Rousselet MC, Konate A, Gallois Y, Ternisien C, Chevailler A, Lunel F: A novel panel of blood markers to assess the degree of liver fibrosis. Hepatology 2005, 42:1373-1381.

20. Colletta C, Smirne C, Fabris C, Toniutto P, Rapetti R, Minisini R, Pirisi M: Value of two noninvasive methods to detect progression of fibrosis among HCV carriers with normal aminotransferases. Hepatology 2005, 42:838-845.

21. Kettaneh A, Marcellin P, Douvin C, Poupon R, Ziol M, Beaugrand M, de Ledinghen $V$ : Features associated with success rate and performance of FibroScan measurements for the diagnosis of cirrhosis in HCV patients: a prospective study of 935 patients. J Hepatol 2007, 46:628-634.

22. Kuno A, Ikehara Y, Tanaka Y, Saito K, Ito K, Tsuruno C, Nagai S, Takahama Y, Mizokami M, Hirabayashi J, Narimatsu H: LecT-Hepa: a triplex lectin-antibody sandwich immunoassay for estimating the progression dynamics of liver fibrosis assisted by a bedside clinical chemistry analyzer and an automated pretreatment machine. Clin Chim Acta 2011, 412:1767-1772.

23. Kuno A, Ikehara Y, Tanaka Y, Angata T, Unno S, Sogabe M, Ozaki H, Ito K, Hirabayashi J, Mizokami M, Narimatsu H: Multilectin assay for detecting fibrosis-specific glyco-alteration by means of lectin microarray. Clin Chem 2011, 57:48-56.

24. Ito $K$, Kuno A, Ikehara $Y$, Sugiyama M, Saito $H$, Aoki $Y$, Matsui $T$, Imamura M, Korenaga M, Murata K, Masaki N, Tanaka Y, Hige S, Izumi N, Kurosaki M, Nishiguchi S, Sakamoto M, Kage $M$, Narimatsu $H_{\text {, }}$ Mizokami M: LecT-Hepa, a glyco-marker derived from multiple lectins, as a predictor of liver fibrosis in chronic hepatitis $C$ patients. Hepatology 2012, 56:1448-1456.

25. Du D, Zhu X, Kuno A, Matsuda A, Tsuruno C, Yu D, Zhang Y, Ikehara Y, Tanaka $Y$, Zhang $X$, Narimatsu H: Comparison of LecT-Hepa and FibroScan for assessment of liver fibrosis in hepatitis B virus infected patients with different ALT levels. Clin Chim Acta 2012, 413:1796-1799.

26. Berzigotti A, Abraldes JG, Tandon P, Erice E, Gilabert R, Garcia-Pagan JC, Bosch J: Ultrasonographic evaluation of liver surface and transient elastography in clinically doubtful cirrhosis. J Hepatol 2010, 52:846-853.

27. Vallet-Pichard A, Mallet V, Nalpas B, Verkarre V, Nalpas A, Dhalluin-Venier V, Fontaine H: FIB-4: an inexpensive and accurate marker of fibrosis in HCV infection. comparison with liver biopsy and fibrotest. Hepatology 2007, 46:32-36.

28. Poordad F, McCone J Jr, Bacon BR, Bruno S, Manns MP, Sulkowski MS, Jacobson IM, Reddy KR, Goodman ZD, Boparai N, DiNubile MJ, Sniukiene V Brass CA, Albrecht JK, Bronowicki JP, SPRINT-2 Investigators: Boceprevir for untreated chronic HCV genotype 1 infection. N Engl J Med 2011, 364:1195-1206

29. Jacobson IM, McHutchison JG, Dusheiko G, Di Bisceglie AM, Reddy KR, Bzowej NH, Marcellin P, Muir AJ, Ferenci P, Flisiak R, George J, Rizzetto M, Shouval D, Sola R, Terg RA, Yoshida EM, Adda N, Bengtsson L, Sankoh AJ, Kieffer TL, George S, Kauffman RS, Zeuzem S, ADVANCE Study Team: Telaprevir for previously untreated chronic hepatitis $C$ virus infection. $N$ Engl J Med 2011, 364:2405-2416.

30. Poordad F, Lawitz E, Kowdley KV, Cohen DE, Podsadecki T, Siggelkow S, Heckaman M, Larsen L, Menon R, Koev G, Tripathi R, Pilot-Matias T, Bernstein B: Exploratory study of oral combination antiviral therapy for hepatitis $C$. N Engl J Med 2013, 368:45-53.

31. Fattovich G, Covolo L, Bibert S, Askarieh G, Lagging M, Clement S, Malerba G, Pasino M, Guido M, Puoti M, Gaeta GB, Santantonio T, Raimondo G, Bruno R, Bochud PY, Donato F, Negro F, ITAHEC Study Group: IL28B polymorphisms, IP-10 and viral load predict virological response to therapy in chronic hepatitis C. Aliment Pharmacol Ther 2011, 33:1162-1172.

32. Giannini E, Risso D, Botta F, Chiarbonello B, Fasoli A, Malfatti F, Romagnoli P, Testa E, Ceppa P, Testa R: Validity and clinical utility of the aspartate aminotransferase-alanine aminotransferase ratio in assessing disease severity and prognosis in patients with hepatitis C virus-related chronic liver disease. Arch Intern Med 2003, 163:218-224.

33. Liu S, Cipriano LE, Holodniy M, Owens DK, Goldhaber-Fiebert JD: New protease inhibitors for the treatment of chronic hepatitis C: a cost-effectiveness analysis. Ann Intern Med 2012, 156:279-290.

34. Lagging M, Romero Al, Westin J, Norkrans G, Dhillon AP, Pawlotsky JM, Zeuzem S, von Wagner M, Negro F, Schalm SW, Haagmans BL, Ferrari C, Missale G, Neumann AU, Verheij-Hart E, Hellstrand K, DITTO-HCV Study Group: IP-10 predicts viral response and therapeutic outcome in difficult-to-treat patients with HCV genotype 1 infection. Hepatology 2006, 44:1617-1625.

35. Mallet $V$, Gilgenkrantz $H$, Serpaggi J, Verkarre $V$, Vallet-Pichard A, Fontaine $\mathrm{H}, \mathrm{Pol} \mathrm{S}$ : Brief communication: the relationship of regression of cirrhosis to outcome in chronic hepatitis C. Ann Intern Med 2008, 149:399-403.

36. Czaja AJ, Carpenter HA: Decreased fibrosis during corticosteroid therapy of autoimmune hepatitis. J Hepatol 2004, 40:646-652.

37. Pearlman BL, Sjogren MH: Treatment options for HCV nonresponders and relapse patients. Gastroenterol Hepatol (N Y) 2010, 6:1-12

38. Shin SR, Sinn DH, Gwak GY, Choi MS, Lee JH, Koh KC, Yoo BC, Paik SW: Risk factors for relapse in chronic hepatitis $C$ patients who have achieved end of treatment response. J Gastroenterol Hepatol 2010, 25:957-963.

39. Backus LI, Boothroyd DB, Phillips BR, Mole LA: Predictors of response of US veterans to treatment for the hepatitis C virus. Hepatology 2007 46:37-47.

40. Tarantino G, Conca P, Sorrentino P, Ariello M: Metabolic factors involved in the therapeutic response of patients with hepatitis $C$ virus-related chronic hepatitis. J Gastroenterol Hepatol 2006, 21:1266-1268.

41. Feld JJ, Hoofnagle $\mathrm{JH}$ : Mechanism of action of interferon and ribavirin in treatment of hepatitis C. Nature 2005, 436:967-972.

42. French D, Watson J, McCahill B, Taggart I, Smith KD: A preliminary evaluation of the functional significance of alpha-1-acid glycoprotein glycosylation on wound healing. Biomed Chromatogr 2002, 16:412-419.

43. Franzblau C, Schmid K, Faris B, Beldekas J, Garvin P, Kagan HM, Baum BJ: The interaction of collagen with alpha1-acid glycoprotein. Biochim Biophys Acta 1976, 427:302-314

44. Goodman ZD, Stoddard AM, Bonkovsky HL, Fontana RJ, Ghany MG, Morgan TR, Wright EC, Brunt EM, Kleiner DE, Shiffman ML, Everson GT, Lindsay KL, Dienstag JL, Morishima C, HALT-C Trial Group: Fibrosis progression in chronic hepatitis C: morphometric image analysis in the HALT-C trial. Hepatology 2009, 50:1738-1749.

45. Marcellin P, Asselah T, Boyer N: Fibrosis and disease progression in hepatitis C. Hepatology 2002, 36:S47-S56.

46. Forns X, Ampurdanes S, Sanchez-Tapias JM, Guilera M, Sans M, SanchezFueyo A, Quinto L, Joya P, Bruguera M, Rodes J: Long-term follow-up of chronic hepatitis $C$ in patients diagnosed at a tertiary-care center. J Hepatol 2001, 35:265-271.

47. Wai CT, Greenson JK, Fontana RJ, Kalbfleisch JD, Marrero JA, Conjeevaram HS, Lok AS: A simple noninvasive index can predict both significant fibrosis and cirrhosis in patients with chronic hepatitis C. Hepatology 2003, 38:518-526.

48. Akobeng AK: Understanding diagnostic tests 3: receiver operating characteristic curves. Acta Paediatr 2007, 96:644-647.

doi:10.1186/1559-0275-11-44

Cite this article as: Zou et al:: LecT-Hepa facilitates estimating treatment outcome during interferon therapy in chronic hepatitis $C$ patients. Clinical Proteomics 2014 11:44. 